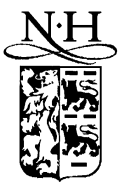

ELSEVIER

\title{
Pattern formation in microdrops of magnetic fluids
}

\author{
I.J. Jang ${ }^{\mathrm{a}}$, H.E. Horng ${ }^{\mathrm{a}, *}$, Y.C. Chiou ${ }^{\mathrm{b}}$, Chin-Yih Hong ${ }^{\mathrm{b}}$, J.M. Wu ${ }^{\mathrm{c}}$, H.C. Yang ${ }^{\mathrm{c}}$ \\ ${ }^{a}$ Department of Physics, National Taiwan Normal University, Taipei 117, Taiwan \\ ${ }^{\mathrm{b}}$ Department of Mechanical Engineering, Da-Yeh University, Chang-Hwa 515, Taiwan \\ ${ }^{\mathrm{c}}$ Department of Physics, National Taiwan University, Taipei 106, Taiwan
}

Received 20 May 1998; received in revised form 1 September 1998

\begin{abstract}
The pattern formation of magnetic fluid films containing magnetic microdrops under applied perpendicular magnetic fields was investigated. At a zero field, these drops of size several $\mu \mathrm{m}$ were initially near-circular. As the field strength increased, these drops changed to dumbbells or branched structure. When the field strength increased again, a labyrinthine structure appeared. At even higher field strength, the connectivity of the drops broke up; and finally an equilibrium hexagonal structure formed inside the film. A new phase transition exists between the labyrinthine structure and the hexagonal structure, which has not been seen before. Also, the reverse process of pattern formation is irreversible. (C) 1999 Elsevier Science B.V. All rights reserved.
\end{abstract}

Keywords: Microdrops; Labyrinthine structure; Hexagonal structure

\section{Introduction}

Due to its rich phenomena, the pattern forming system of magnetic fluid films subjected to an external magnetic field perpendicular to the plane of films has recently been under extensive study [1-3]. When a magnetic fluid thin film is subjected to a perpendicular magnetic field, the particles in the film start to agglomerate and form particle columns. As the field strength increases, the columns evolve from a disordered column phase to a hexagonal structure, and then to a labyrinthine pattern [3]. In actual applications, it often happens that the

\footnotetext{
* Corresponding author. Fax: + 886-2-29309439.

E-mail address: phyfv001@scc.ntnu.edu.tw (H.E. Horng)
}

carrier of magnetic fluid escapes from the film and the fluid inside the film becomes over-saturated. Some of the particles will then condense out of the fluid and form microdrops inside the film. In order to understand the effect of this consequence to the applications, we study the pattern formation of an over-saturated magnetic microdrop system.

The pattern forming system of magnetic fluid drops have been investigated. Tsebers et al. first reported that the original cylindrical drop evolves to a dumbbell and bends as the field strength over some threshold. Over another threshold, the dumbbell becomes labyrinthine [4]. Langer et al. suggested that the pattern formation depends on initial conditions [5]. Dickstein et al. found that the degree of branching of the initially circular shapes depends not only on the magnetic field but also on 


$$
\mathrm{dH} / \mathrm{dt}=5 \mathrm{Oe} / \mathrm{s}
$$

(a) $\mathrm{H}=0$

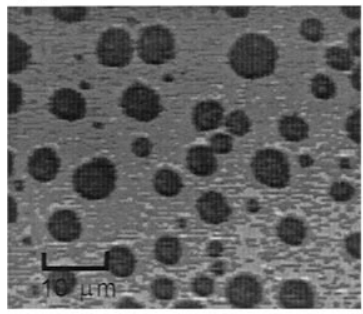

(c) $\mathrm{H}=65 \mathrm{Oe}$

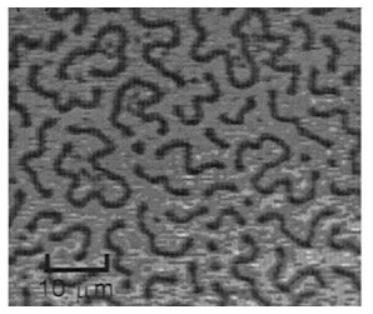

(e) $\mathrm{H}=100 \mathrm{Oe}$

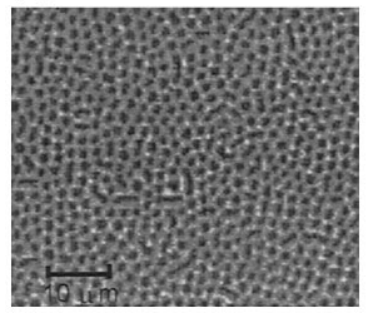

(b) $\mathrm{H}=50 \mathrm{Oe}$

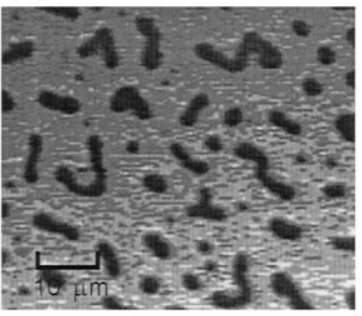

(d) $\mathrm{H}=90 \mathrm{Oe}$

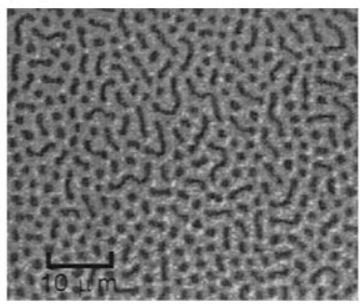

(f) $\mathrm{H}=250 \mathrm{Oe}$

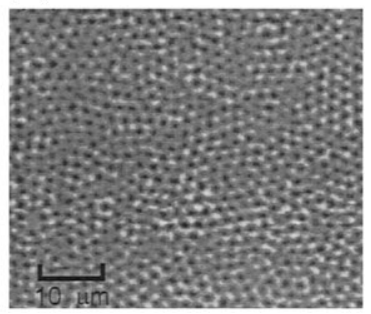

Fig. 1. Evolution of pattern formation of magnetic fluid thin film containing magnetic microdrops subjected to a perpendicular magnetic field at low sweep rate of $5 \mathrm{Oe} / \mathrm{s}$.

the rate of change of magnetic field strength [6]. The last two all indicated that the branched structure spreads without any further change in connectivity. This implies that the labyrinthine is a final state of a magnetic fluid drop under a magnetic field.

\section{Experimental details}

The kerosene-based magnetic fluid used in this study was prepared by the co-precipitation technique. To form a ferrofluid thin film, the saturated magnetic fluid was injected into a $4 \mathrm{~cm} \times 1 \mathrm{~cm}$ rectangular glass cell of thickness $10 \mu \mathrm{m}$ and kept at

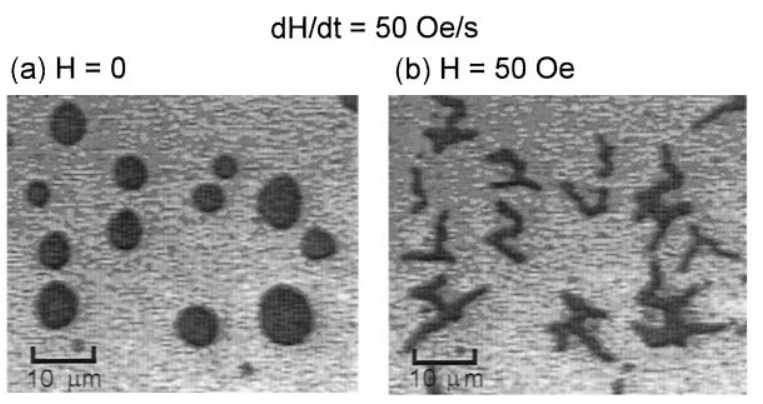

(c) $\mathrm{H}=55 \mathrm{Oe}$

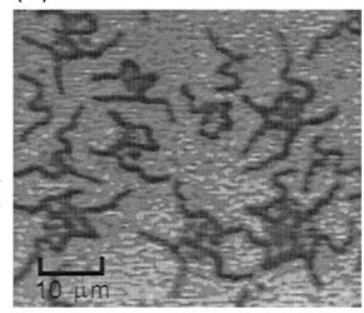

(d) $\mathrm{H}=65 \mathrm{Oe}$

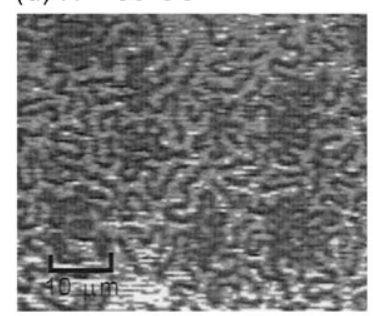

(e) $\mathrm{H}=90 \mathrm{Oe}$

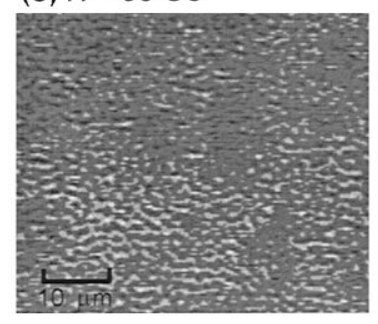

(f) $\mathrm{H}=250 \mathrm{Oe}$

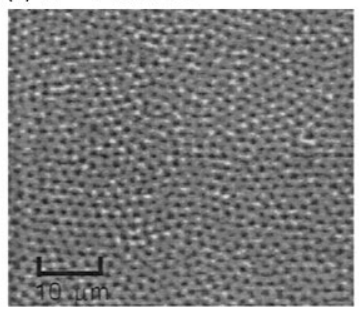

Fig. 2. Evolution of pattern formation of magnetic fluid thin film containing magnetic microdrops subjected to a perpendicular magnetic field at high sweep rate of $50 \mathrm{Oe} / \mathrm{s}$.

room temperature. Due to slight evaporation of the kerosene carrier from the glass cell, the magnetic fluid became over-saturated after 2 days and then the disordered microdrops were formed inside the magnetic fluid thin film.

Throughout this experiment, the magnetic fields were generated by a pair of solenoids. The applied magnetic field was uniform; deviations of the field strength in the region of the cell were found to be less than $1 \%$. To study the pattern formation of magnetic fluid containing these microdrops inside the film, photo images of the structure patterns were taken by using a Leitz optical microscope and recorded in a personal computer through a CCD video camera. 


\section{Results and discussion}

In this paper, our attention is focused on the shape evolution of microdrops as a result of the application of a magnetic field as well as the field sweep rate. Figs. 1 and 2 show the evolution process of microdrops in an over-saturated magnetic fluid thin film subjected to a perpendicular magnetic field. At a zero field, these drops of size several $\mu \mathrm{m}$ are initially near-circular (Fig. 1a and Fig. 2a). Upon the application of a magnetic field, the competition between the surface tension and the magnetic dipole interactions occurs. The circular drops evolve quickly to dumbbells (Fig. 1b) or branched structure (Fig. 2b), depending on the field sweep rate which is in agreement with the results as suggested by Dickstein [6]. At a lower sweep rate, magnetic particles within the drops are allowed to rearrange in such a way that a dumbbell shape can be formed. As the field strength increases, the dumbbells elongate and bend (Fig. 1c). At a higher field strength, a labyrinthine structure forms in the film (Fig. 1d). If the field strength is even higher, the labyrinthine structure becomes an equilibrium hexagonal structure (Fig. 1e and f). On the other hand, at a higher sweep rate, these drops evolve to a branched structure (Fig. 2b and c), to a labyrinthine pattern (Fig. 2d and e) and finally to an equilibrium hexagonal structure (Fig. 2f). It is suggested that there is a critical sweep rate (around $10-20 \mathrm{Oe} / \mathrm{s}$ in our system) between the formation of dumbbell and branched structure. It must be noted that the labyrinthine structure is indeed an intermediate state; this disagrees with the results given by prior reports [4-6]. The cause of the pattern formation is believed to be mainly due to the competition between surface tension, the magnetic dipole interactions and the attractive force due to the external

\section{Reverse process $(\mathrm{dH} / \mathrm{dt}=-5 \mathrm{Oe} / \mathrm{s})$}

(a) $\mathrm{H}=65 \mathrm{Oe}$

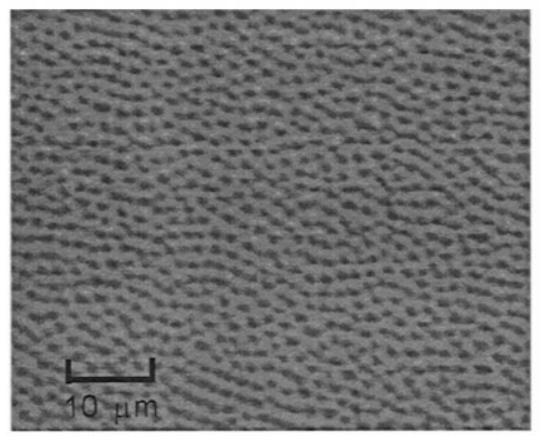

(c) $\mathrm{H}=0, \mathrm{t}=0 \mathrm{~min}$.

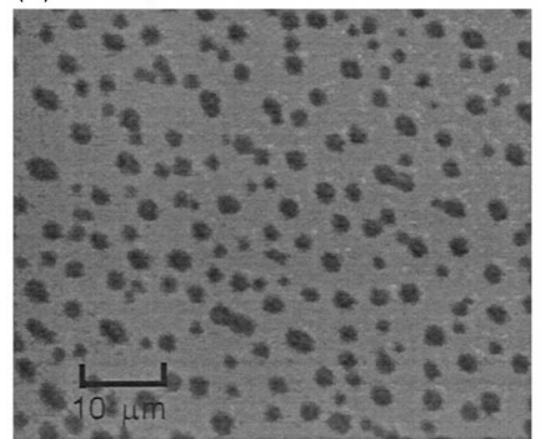

(b) $\mathrm{H}=50 \mathrm{Oe}$

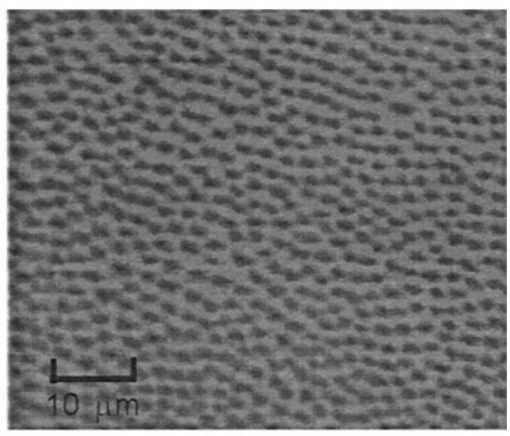

(d) $\mathrm{H}=0, \mathrm{t}=10 \mathrm{~min}$.

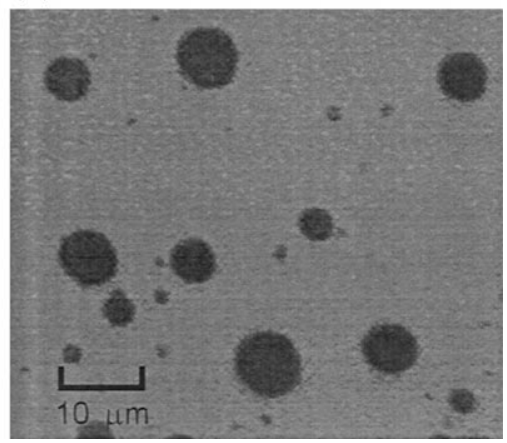

Fig. 3. The reverse process of pattern formation of magnetic fluid thin film containing magnetic microdrops subjected to a perpendicular magnetic field at sweep rate of $-5 \mathrm{Oe} / \mathrm{s}$. 
magnetic field. At the initial circular shape phase, the surface tension dominates. From the labyrinthine pattern to hexagonal structures, the magnetic dipole interactions dominate the surface tension resulting in the breakup of connectivity of dumbbells or branched structure in labyrinthine phase. This breakup repeats until the system reaches an equilibrium state; and the system becomes hexagonal in which the repulsive force due to dipole interaction and the attractive force due to external magnetic field dominate. Sweeping the field back to the zero field state, the system restores the shape to its initial circular form without going through the labyrinthine pattern, dumbbells and/or branched structure. The system changes from a hexagonal structure to a disordered column phase and then to the initial circular form. Thus, the process of the pattern formation in microdrops of magnetic fluid is irreversible. Fig. 3 shows the reverse process with a sweep rate of $-5 \mathrm{Oe} / \mathrm{s}$.

\section{Conclusion}

In this work, we first observed that the labyrinthine structure becomes an equilibrium hexagonal structure in a magnetic microdrop system of oversaturated magnetic fluid films subjected to perpendicular magnetic fields. The pattern formation of magnetic microdrop system started from an initially circular shape to a dumbbell phase or branched structure. A critical field sweep rate exists. A dumbbell phase occurs when the field sweep rate is below this value; otherwise a branched structure forms. As the field strength increases, the pattern changes to a labyrinthine structure and finally to an equilibrium hexagonal structure. Moreover, the pattern formation is an irreversible process.

\section{References}

[1] J. Liu, E.M. Lawrence, A. Wu, M.L. Ivey, G.A. Flores, K. Javier, J. Bibette, J. Richard, Phys. Rev. Lett. 74 (1995) 2828.

[2] A.T. Skjeliorp, Phys. Rev. Lett. 51 (1983) 2306.

[3] Chin-Yih Hong, I.J. Jang, H.E. Horng, C.J. Hsu, Y.D. Yao, H.C. Yang, J. Appl. Phys. 81 (1997) 4275.

[4] A.O. Tsebers, M.M. Maiorov, Sov. Tech. Phys. Lett. 6 (1980) 50.

[5] S.A. Langer, R.E. Goldstein, D.P. Jackson, Phys. Rev. A 46 (1992) 4894.

[6] A.J. Dickstein, S. Erramilli, R.E. Goldstein, D.P. Jackson, S.A. Langer, Science 261 (1993) 1012. 